\title{
The members' enquiry service: frequently asked questions
}

\author{
Lisa A Allerton, Gillian Penney
}

\section{Background}

Lamotrigine is an anti-epileptic drug used in the management of adults with simple or complex partial seizures or with primary or secondary generalised tonicclonic seizures. ${ }^{1}$ In June 2005, GlaxoSmithKline advised clinicians of important changes to the prescribing information for lamotrigine (Lamictal $\left.{ }^{\circledR}\right){ }^{2}$ Concerns were raised about the possibility of a drug interaction between lamotrigine and hormonal contraception. As a result, the Clinical Effectiveness Unit (CEU) received several enquiries pertaining to this issue and reviewed the current evidence.

\section{Illustrative CEU response \\ Clinical question}

For women who are taking lamotrigine, are there any interactions that should be considered if hormonal contraception is used?

\section{Summary of response}

Based on current evidence the CEU suggests the following:

- Small studies have shown no evidence of ovulation when lamotrigine was used with combined oral contraception. Evidence on progestogen-only contraceptives is unavailable. There is no evidence that lamotrigine is a liver enzyme-inducing drug.

- The possibility of drug interactions should be considered and discussed when prescribing any medicines to women of reproductive age.

- The benefits and risks of all contraceptive methods (hormonal and non-hormonal) should be considered. After counselling, women using lamotrigine may choose to continue with hormonal contraception.

- Women using lamotrigine should be advised that seizure frequency may increase when initiating combined oral contraception and lamotrigine side effects may increase when discontinuing combined oral contraception.

The CEU recommends that clinicians should monitor any clinical effects when initiating or discontinuing combined oral contraception in women using lamotrigine. There are no data concerning progestogen-only contraceptive methods and drug interactions with lamotrigine. Moreover, there is no evidence to suggest that lamotrigine is a liver enzyme-inducing drug. Evidence does not support the advice that women using lamotrigine should use only non-hormonal contraceptive methods.

Evidence-based medicine question (which guided our literature search strategy)

Population: Women taking lamotrigine.

Intervention: Hormonal contraception.

Outcome: Interactions and efficacy.

\section{J Fam Plann Reprod Health Care 2006; 32(1): 43-44}

Clinical Effectiveness Unit, Faculty of Family Planning and Reproductive Health Care, London, UK

Lisa A Allerton, BSc, MSc, Research Assistant

Gillian Penney, FRCOG, MFFP, Honorary Director

Correspondence to: Ms Lisa Allerton, FFP CEU, Office 63, Aberdeen Maternity Hospital, Cornhill Road, Aberdeen AB25 2ZD, UK. Tel: +44 (0) 1224 553623. E-mail: ffp.ceu@abdn.ac.uk
Table 1 Sources used in developing the Member's Enquiry Response

\begin{tabular}{ll}
\hline Source searched & Information identified \\
\hline The National Guidelines Clearing House & No relevant information \\
Existing FFPRHC and RCOG Guidance & See text \\
WHO publications: Medical Eligibility & No relevant information \\
Criteria for Contraceptive Use (2004) & \\
and Selected Practice Recommendations for & \\
$\begin{array}{l}\text { Contraceptive Use (2005) } \\
\text { The Cochrane Library }\end{array}$ & No relevant information \\
MEDLINE and EMBASE from 1996 to 2005 & See text \\
\hline
\end{tabular}

FFPRHC, Faculty of Family Planning and Reproductive Health Care; RCOG, Royal College of Obstetricians and Gynaecologists; WHO, World Health Organization.

\section{Information sources}

The CEU searched the sources listed in Table 1 in developing this Member's Enquiry Response

\section{Evidence reviewed: MEDLINE and EMBASE}

The effect of hormonal contraception on the clinical action of lamotrigine. A small case series highlighted a possible drug interaction between lamotrigine and two combined oral contraceptives (COCs) containing $20 \mu \mathrm{g}$ or $30 \mu \mathrm{g}$ ethinylestradiol with desogestrel. ${ }^{3}$ Four women had an increase in frequency of seizures and a decrease in serum lamotrigine concentrations when COC was initiated. One woman reported an increase in lamotrigine side effects (double vision, nausea, dizziness) when COC was stopped; lamotrigine levels increased after discontinuation of COC.

In 2003, a small prospective study (56 women) investigated the possible drug interaction between lamotrigine and COCs containing ethinylestradiol/ levonorgestrel or ethinylestradiol/desogestrel. ${ }^{4}$ This study found that the bioavailability of lamotrigine was apparently reduced with COC use. Mean plasma levels were significantly lower $(p<0.0001)$ in COC users than in nonCOC users [i.e. 13 (range, 2-29) $\mu \mathrm{mol} / \mathrm{l}$ cf. 28 (range, 7-69) $\mu \mathrm{mol} / 1]$. However, the clinical effect on seizure frequency was not reported.

Another small prospective study looking at 16 women using lamotrigine (300 mg/day) for bipolar disorder found that lamotrigine clearance increased by approximately twofold with concomitant COC use. ${ }^{5}$ Also, the maximum concentration $\left(C_{\max }\right)$ of lamotrigine was reduced on average by $39 \%$.

One small study investigated the potential interaction between lamotrigine and the combined vaginal ring. Lamotrigine concentrations were reduced by $15-50 \%$ in women using the vaginal ring (ethinylestradiol/ etonogestrel). ${ }^{6}$

The effect of lamotrigine on the efficacy of hormonal contraception. In the prospective study with 16 women using lamotrigine for bipolar disorder ${ }^{5}$ it was concluded that the bioavailability of ethinylestradiol remained unchanged but the bioavailability of levonorgestrel was reduced. However, the reduction in levonorgestrel was not significant. The authors reported that there was no evidence to suggest that ovulation (as measured by serum progestogen concentration) had occurred and contraceptive efficacy was unlikely to have been affected.

In addition, the authors concluded that pre-dose serum lamotrigine concentrations increased in a fairly rapid and 


\section{FROM THE CEU/NEWS ROUNDUP}

linear manner during the 'pill-free' week, with concentrations at the end of the week being, on average, approximately two-fold higher than during $\mathrm{COC}$ coadministration. The clinical effects of this were not reported in the study; however, the CEU sought guidance from several physicians/neurologists. All were in agreement that that the degree of fluctuation in lamotrigine levels seen in the 'pill-free' week is unlikely to be of clinical significance. They confirmed that serum levels are unhelpful in predicting therapeutic effects or side effects.

No evidence was found with respect to progestogenonly methods. Moreover, there is no evidence to suggest that lamotrigine is a liver enzyme-inducing drug. Evidence does not support the advice that women using lamotrigine should use only non-hormonal methods.

\section{Evidence reviewed: existing FFPRHC Guidance}

The Faculty of Family Planning and Reproductive Health Care (FFPRHC) have produced a CEU Faculty Statement on the changes to prescribing information for lamotrigine. ${ }^{7}$

\section{Disclaimer}

The advice given in this Member's Enquiry Response has been prepared by the FFPRHC Clinical Effectiveness Unit team. It is based on a structured search and review of published evidence available at the time of preparation. The advice given here should be considered as guidance only. Adherence to it will not ensure a successful outcome in every case and it may not include all acceptable methods of care aimed at achieving the same result. This response has been prepared as a service to FFPRHC members, but is not an official Faculty Guidance product; Faculty Guidance is produced by a different and lengthier process. It is not intended to be construed or to serve as a standard of medical care. Such standards are determined on the basis of all clinical data available for an individual case and are subject to change as scientific knowledge advances. Members are welcome to reproduce this response by photocopying or other means, in order to share the information with colleagues.

References

1 British National Formulary, vol. 49. London, UK: British Medical Association and the Royal Pharmaceutical Society of Great Britain, March 2005. http://www.BNF.org [Accessed 25 August 2005].

2 GlaxoSmithKline UK. GlaxoSmithKline Health Care Update: Lamictal (Lamotrigine). 2005. http://www.gsk.com. [Accessed 25 August 2005].

3 Sabers A, Buchholt JM, Uldall P, Hansen EL. Lamotrigine plasma levels reduced by oral contraceptives. Epilepsy Res 2001; 47: 151-154.

4 Feskanich D, Hunter DJ, Willett WC, Spiegelman D, Stampfer MJ, Speizer FE, et al. Oral contraceptive use and risk of melanoma in premenopausal women. Br J Cancer 1999; 81: 918-923.

5 Sidhu J, Job S, Buisara S, Phillipson R. Pharmacokinetics and Hormonal Effects of Lamotrigine-Combined Oral Contraceptive Coadministration. 2005. http://ctr.gsk.co.uk/Summary/lamotrigine/ 1_LAM10016.pdf [Accessed 25 August 2005].

6 Stodieck SRG, Schwenkhagen AM. Lamotrigine (Letter). Epilepsia 2004; 45(Suppl. 7)

7 Faculty of Family Planning and Reproductive Health Care Clinical Effectiveness Unit. Faculty Statement from the CEU on Changes to Prescribing Information for Lamotrigine. 2005. http://www. ffprhc.org.uk/admin/uploads/lamotrigine.pdf [Accessed 24 October 2005].

\section{News Roundup}

\section{Reporting of sexually active young teenagers}

In the wake of the Bichard enquiry, a review of the current guidance, Working Together to Safeguard Children, is taking place. It is due to be published in January 2006 but it seems there is already some confusion regarding reporting of underage sex. Child protection committees have already issued guidance which conflicts with the current Department of Health advice. Presently the Department of Health recognises that children younger than 16 years have rights to confidentiality if considered competent. However, Sheffield and London child protection committees have produced protocols that compel health professionals to report all sexual activity in young people under the age of 13 years, similar to the situation in the USA and some states in Australia. In a country with the highest teenage pregnancy rate in Europe, I wonder how teenagers will view this new guidance, and how it will affect vulnerable teenagers in our society.

\section{Reference \\ Bastable R, Sheather J. Mandatory reporting to the police of} all sexually active under-13s. BMJ 2005; 331: 918-919.

Reported by Laura Patterson, MRCGP, DFFP $G P$, Cirencester, $U K$

\section{Tackling chlamydia}

A team of scientists at the University of Cambridge has developed a new test for chlamydia. It is simple to use, cheap, takes less than 25 minutes to give a result and can be used with non-invasive samples. At present a performance evaluation study is being undertaken at Brook in Birmingham, UK. Following this it will be licensed and made available for use by health professionals. Preliminary testing results are looking very encouraging. Boots the Chemist are also attempting to make chlamydia testing accessible. From November 2005, branches of Boots the Chemist across London, UK will undertake a pilot programme offering testing and treating of chlamydia. This is another valuable tool to complement the continuing development of the national screening programme.

Source: Department of Health

Reported by Laura Patterson, MRCGP, DFFP GP, Cirencester, UK

\section{NICE guidance for LARC}

The National Institute for Health and Clinical Excellence (NICE) guideline for long-acting reversible contraception (LARC) is 40 pages in length. It sets clear guidance for the use of the four methods of LARC available in the UK, namely copper intrauterine devices (IUDs), the intrauterine system (IUS), progesterone-only injectable contraception and progesterone-only subdermal implants. However, it does remind us that most of the evidence is extrapolated, as there is little available robust evidence in this area. It reminds us about good communication and encourages us to help women make an informed choice about their contraception. At present the uptake of these forms of contraception is low. However, LARC could reduce the rate of unplanned pregnancies. Bearing in mind that these methods are more cost effective than the combined oral contraceptive at 1 year, this document is a valuable reminder to health care professionals guiding women in their choice.

The individual sections of guidance are pretty much as one would expect. I was, however, surprised by the quoted figures cited for discontinuation rates. Up to $50 \%$ of women stop using IUDs within 5 years, most commonly because of unacceptable vaginal bleeding and pain. Up to $60 \%$ of women stop using the IUS within 5 years, again because of unacceptable vaginal bleeding and pain. Up to $50 \%$ of women stop using depot medroxyprogesterone acetate
(DMPA) for reasons of altered bleeding pattern. This clearly emphasises the importance of giving clear information and helping women make an informed choice.

Without robust evidence, NICE indicate that DMPA may be given up to 2 weeks late without the need for additional contraceptives. This is outside the licensed indications. It also makes reference to the use of the subdermal implant Implanon ${ }^{\circledR}$ in women weighing over $70 \mathrm{~kg}$. However, there is no recommendation as to whether there may be reduced effectiveness in the final year of use in this group.

The guidance is due for review in 4 years' time but I am sure will provide an extremely useful reference for health professionals.

Source: www.nice.org.uk

Reported by Laura Patterson, MRCGP, DFFP GP, Cirencester, UK

\section{Sex education in the USA}

There is a substantial federal budget available in the USA for sex education. Several states recently have rejected this money for fear that the education they are now expected to provide has become very restrictive. Since 1996, the state of Maine has been using 'a comprehensive approach to family life' education. Through this they have achieved a steep decline in teenage pregnancies and in the rate of terminations. However, the federal programme now requires teachers to advocate abstinence until marriage and mutual faithfulness afterwards rather than comprehensive sex education. They must emphasise that abstinence is the only certain way to avoid pregnancy and sexually transmitted infections.

Reference

Tanne JH. US state rejects deferral funding for abstinence only sex education $B M J 2005 ; 331: 715$.

Reported by Laura Patterson, MRCGP, DFFP GP, Cirencester, UK 